\title{
Noether Currents for Bosonic Branes
}

\author{
G. Arreaga( ${ }^{(1)}$, R. Capovilla( ${ }^{(1)}$ and J. Guven ${ }^{(2)}$ \\ (1) Departamento de Física \\ Centro de Investigación y de Estudios Avanzados del IPN \\ Apdo. Postal 14-740,07000 México, DF, MEXICO \\ (2) Instituto de Ciencias Nucleares \\ Universidad Nacional Autónoma de México \\ Apdo. Postal 70-543, 04510 México, DF, MEXICO
}

October 29, 2018

\begin{abstract}
We consider a relativistic brane propagating in Minkowski spacetime described by any action which is local in its worldvolume geometry. We examine the conservation laws associated with the Poincaré symmetry of the background from a worldvolume geometrical point of the view. These laws are exploited to explore the structure of the equations of motion. General expressions are provided for both the linear and angular momentum for any action depending on the worldvolume extrinsic curvature. The conservation laws are examined in perturbation theory. It is shown how nontrivial solutions with vanishing energy-momentum can be constructed in higher order theories. Finally, subtleties associated with boundary terms are examined in the context of the brane Einstein-Hilbert action.
\end{abstract}




\section{Introduction}

A brane is a relativistic extended object propagating in a background spacetime, usually treated as fixed, of some given dimension $N$. The spacetime trajectory of the brane is a timelike worldvolume of some lower dimension $D$ : for a relativistic string $D=2$; for a domain wall, or membrane, $D=3$, and so on. The dynamics of the brane is described by some local action constructed using scalars that characterize the geometry of the worldvolume. The possibilities are limited by worldvolume reparameterization invariance, ambient spacetime diffeomorphism invariance, and, when $D<N-1$, invariance under rotations in the $N-D$ dimensional plane normal to the worldvolume. The simplest action of this kind is the Dirac-Nambu-Goto [DNG] action, proportional to the volume swept out by the brane in the course of its evolution. This action depends only on first order derivatives of the field variables, the embedding functions that define the worldvolume. Originally proposed by Dirac in the context of an extensible model for the electron $(D=3)$ [1, 2], it was later exploited by Nambu and Goto for the case of a relativistic string $(D=2)$ to model hadronic matter [3]. It constitutes the point of departure in the construction of modern string theory [4]. Higher dimensional $(D>1)$ DNG branes also play an increasingly important role as solitonic solutions of this theory (see e.g. [5, 6]).

Despite significant developments in string theory, it would appear fair to claim that a comprehensive understanding of the dynamics of relativistic extended objects is still lacking. In particular, there are many contexts where the DNG action is clearly inadequate, and it has been necesssary to consider a more general action that includes higher-order geometrical scalars which depend on the curvature of the worldvolume; possibly even its derivatives. These additions do modify the dynamics substantially. The lowest order correction to the dynamics of a purely geometrical object is described by an action quadratic in the extrinsic curvature. For example, in a phenomenological approach, the addition of such a 'rigidity' term to the DNG action was proposed in the eighties by Polyakov, and independently by Kleinert, as an improved effective action for QCD [7]. Earlier still, hamiltonians of this form were studied in condensed matter physics as models to describe the mechanical properties of lipid membranes [8]. Higher-order corrections can also arise in systematic approximations. For example, in the context of the physics of topological defects, curvature terms are induced by considering an expansion in the thickness of the defect [9]. The addition of such terms typically associates an energy penalty with the formation of a spike. These corrections thus become important on short distance scales serving to smooth out the curvature singularities which will arise in the course of the DNG dynamics. In Ref. [10], an effective action of this form was obtained explicitly for domain walls by integrating out the microscopic degrees of freedom of the underlying field theory which vary rapidly on the length scale of the wall. In the case of strings it has been argued that the lowest order effective action which describes the behavior in the neighborhood of a cusp must contain terms which are of fourth order in the extrinsic curvature of the worldvolume [11].

Another, different, source of higher-order terms comes from supersymmetric branes. 
They arise once an effective action is obtained by integrating out the fermionic degrees of freedom [12, 13, 14].

In this paper, we explore the structure of the conservation laws associated with the Poincaré invariance of a local brane action propagating in Minkowski spacetime. These occur as a consequence of the induced internal symmetries on the field theory described by the embedding of the brane worldvolume in spacetime.

At one level, the determination of the associated conserved quantities is straightforward. One can write the action explicitly in terms of the embedding functions, perhaps in terms of a natural parametrization, if available. Noether's theorem then generates the conserved momentum and angular momentum. This procedure is perfectly adequate for a DNG object where the lagrangian is a function of the intrinsic geometry alone (see, for example, [15] and [4] for a DNG string, or [16] for higher dimensional DNG objects.) If, however, the geometrical action involves either the intrinsic or the extrinsic curvature, so that the action depends on second derivatives of the embedding functions or higher, the manifest covariance of the geometrical action we start out with gets mutilated in this straighforward approach, and the conserved quantities do not possess any obvious geometrical form.

There are several ways to remedy this shortcoming. One way exploits the elegant formalism developed by Iyer and Wald for diffeomorphism invariant theories [17]. This involves grouping higher derivative terms into totally symmetric combinations, and obtaining the general expressions for the Noether charges. For the case of branes, however, this procedure does not result in the natural geometric quantities of the worldvolume, and the translation is ackward. An alternative strategy is to perform a hamiltonian analysis. This is certainly necessary if one is interested in the canonical quantization of the theory. However, since the extrinsic curvature of a brane can be considered, roughly, as a generalization to higher dimensional objects of the acceleration of a relativistic particle, one is dealing with the complicated hamiltonian analysis of a higher derivative theory. Another approach which is spacetime manifestly covariant was pioneered by Carter (see e.g. 18 and references therein). It focuses on the derivation of the stress-energy tensor for the theory, and the conserved quantities are constructed by contraction with the appropriate background Killing vector fields. This approach is certainly convenient when external fields are present, but, in our opinion, it does not take full advantage of the natural geometric structures on the worldvolume.

In this paper we develop an independent approach, close in spirit to the one developed by Carter, but differing in our emphasis on the worldvolume geometry. We exploit the geometrical formalism introduced by two of the authors in [19], tailored to the worldvolume of the extended object, to describe deformations of the worldvolume to express the variations in the worldvolume geometry induced by a Poincaré transformation in a covariant way. This will permit us to write down the corresponding conservation laws in a geometrical form adapted to the worldvolume.

Following Carter, we express the equations of motion in terms of the conservation of the linear momentum. However, we go one step further by separating these equations into two sets, obtained by projecting the local form of the conservation law onto and normal 
to the worldvolume. In doing this, we necessarily dismantle the worldvolume divergence appearing in the conservation law, which one might consider a step in the wrong direction. The advantage, however, is that the $N-D$ normal projections encode completely the worldvolume diffeomorphism invariant content of the former equations. The associated gauge redundancy in the conservation laws is captured in the $D$ tangential projections or Bianchi identities. When we cast the conservation law this way, the distinct dynamical roles played by the tangential and normal projections of the linear momentum density in the theory also become explicit. It is then an easy matter to identify characteristics of the projections associated with a specific theory, such as, for example, the conditions under which a theory is conformally invariant.

We demonstrate how this structure can be exploited in perturbation theory. In particular, we exploit the linearization of the conservation law to provide a novel derivation of the linearized equations of motion, which, in addition to its technical merits, also throws light on the underlying structure.

We also examine angular momentum conservation. The structure of the spin for a DNG action has a very special form. We demonstrate explicitly how new structures enter when actions of higher order are considered. We point out that the Regge inequality for string theory continues to hold along extremal solutions of certain higher order theories.

An important issue in the path integral formulation of the quantum theory is the identification of appropriate boundary conditions to be imposed on the initial and final configurations in the variational principle. These boundary conditions are largely a matter of choice in the classical theory. An example is provided by the brane Einstein-Hilbert action 20, 21] which involves terms linear in second derivatives of the embedding functions, so that the equations of motion, in common with DNG theory, is also second order in derivatives. A spurious surface term occurs in the variational principle which signals problems in the path integral quantization of the theory. We provide a geometric approach to analysing this problem which is guided by the analogous problem in General Relativity [22, 23].

It should be mentioned that various special cases the Noether currents of particular branes have been analyzed in the literature, using different approaches. For example, Letelier has considered a DNG membrane $(D=2)$ propagating in four-dimensional Minkowski spacetime, with the addition of a term porportional to the worldvolume intrinsic curvature, the brane Einstein-Hilbert action [24]. A relativistic string with arbitrary curvature corrections has been studied by Boisseau and Letelier [25]. The more general case of a brane action at most quadratic in the extrinsic curvature in an arbitrary background spacetime is the subject of a detailed analysis by Carter in [26]. For an alternative treatment of extrinsic curvature actions which uses the language of differential forms, see Hartley and Tucker in [27]. We find complete agreement with their treatment, once details of notation have been taken into account.

As is well known, the expressions for the momenta themselves are ambigous. One is always free to add a total divergence to the lagrangian, which contributes to the momenta without affecting the dynamics. Moreover, one can add a term that is identically conserved 
to the momentum densities. We will keep in mind this freedom, and we will have occasion to use it for example in the specific case of the brane Einstein-Hilbert action, but we will not develop the general formalism to treat it in full generality. For the interested reader, this subject and its consequences on the global structure of the space of solutions of a relativistic theory is developed in detail e.g. by Anderson and Torre in [28, 29, 30].

This paper is organized as follows. In the next section, we define the linear and angular momentum for a general brane. In Sect. 3, we show how the equations of motion can be expressed in terms of the conservation of the linear momentum. The familiar case of a DNG brane is the subject of Sect. 4, which serves as a useful illustration of the formalism. In Sect. 5, we move on to the general case of a rigid lagrangian depending on the extrinsic curvature of the worldvolume. We obtain both the linear and angular momentum for this class of theories. In order to make our treatment more concrete we specialize to low order specific examples in Sect. 6, including the Einstein-Hilbert action for a brane. In Sect. 7, we provide a novel approach to the linearized equations of motion, in terms of the linearization of the linear momentum density. The introduction of a surface term of the York type in the brane Einstein-Hilbert action is the subject of Sect. 8. We conclude in Sect. 9 with a brief discussion. Finally, in an appendix, we sketch the extension of the formalism to lagrangians that depend on derivatives of the extrinsic curvature.

\section{Conservation laws}

Let us consider a brane, of dimension $D-1$, propagating in a fixed background Minkowski spacetime of dimension $N$. For simplicity, we suppose that this object is either infinite in extent, or closed. In the case of an infinite object, we will assume in the following that appropriate fall-off conditions are chosen on the fields, so that the formal expressions we derive actually exist. The extension of the formalism to objects with finite (timelike) boundaries, or with loaded edges, relevant for the treatment of hybrid branes, such as a string with monopoles at its edges, or a wall bounded by strings (see e.g. [31]), is straightforward, and will not be considered in this paper.

The domain of integration is the timelike worldvolume $m$, given by the embedding functions

$$
x^{\mu}=Y^{\mu}\left(\xi^{a}\right)
$$

where $x^{\mu}$ are local coordinates in the ambient Minkowski spacetime, and $\xi^{a}$ local coordinates for the worldvolume $(\mu, \nu, \cdots=0,1, \cdots N-1$, and $a, b, \cdots=0,1 \cdots, D-1)$. The worldvolume is described by the evolution of a $D$-1-dimensional brane between fixed initial and final (spacelike) configurations.

The initial and final configurations are described completely by two spacelike hyper-

surfaces on the worldvolume $m, \Sigma_{(i)}$ and $\Sigma_{(f)}$. For definiteness, we parametrize these hypersurfaces by the embedding functions,

$$
\xi^{a}=X_{(i)}^{a}\left(u^{A}\right)
$$




$$
\xi^{a}=X_{(f)}^{a}\left(u^{A}\right)
$$

respectively, where $u^{A}$ are local coordinates for the spacelike hypersurfaces $(A, B, \cdots=$ $1, \cdots, D-1)$.

It turns out that it is also convenient to consider them as embedded directly in Minkowski spacetime, via map composition, so that e.g. with (11) and (2) we have

$$
x^{\mu}=X_{(i)}^{\mu}\left(u^{A}\right)=e_{a}^{\mu} X_{(i)}^{a}\left(u^{A}\right),
$$

where we denote by

$$
e^{\mu}{ }_{a}=\frac{\partial Y^{\mu}}{\partial \xi^{a}}
$$

the $D$ tangent vectors to the worldvolume $m$. We will also use

$$
\epsilon_{A}^{\mu}=\frac{\partial X^{\mu}}{\partial u^{A}},
$$

to denote the $D-1$ tangent vectors to $\Sigma_{(i)}$ or $\Sigma_{(f)}$ as embedded in spacetime.

The union of these two hypersurfaces is the boundary $\partial m$ of the worldvolume $m$, with the understanding that the natural orientation of the initial hypersurface $\Sigma_{(i)}$ is opposite to that of $\Sigma_{(f)}$. We consider only oriented branes.

We now make an infinitesimal deformation of the embedding functions for the worldvolume $m$,

$$
Y^{\mu}(\xi) \rightarrow Y^{\mu}(\xi)+\delta Y^{\mu}(\xi)
$$

This displacement can be seen as a diffeomorphism of the ambient spacetime, and it will induce a deformation of the worldvolume geometry.

We decompose an arbitrary infinitesimal deformation of the embedding $\delta Y^{\mu}$ into its parts tangential and normal to the worldvolume,

$$
\delta Y^{\mu}=\Phi^{a} e^{\mu}{ }_{a}+\Phi^{i} n^{\mu}{ }_{i},
$$

where the tangent vectors $e^{\mu}{ }_{a}$ have been defined earlier, by Eq. (5), and $n^{\mu}{ }_{i}$ are the $N-D$ vectors normal to the worldvolume $m(i, j, \cdots=1,2, \cdots, N-D)$. These are defined by

$$
\eta_{\mu \nu} e^{\mu}{ }_{a} n_{i}^{\nu}=0
$$

where $\eta_{\mu \nu}$ is the Minkowski metric, with only one minus sign, which will be used to raise and lower spacetime indices. We choose to normalize the normal vector fields as

$$
\eta_{\mu \nu} n^{\mu}{ }_{i} n^{\nu}{ }_{j}=\delta_{i j}
$$

where $\delta_{i j}$ is the Kronecker delta.

The worldvolume geometry is described completely by the induced metric $\gamma_{a b}$, the extrinsic curvature $K_{a b}{ }^{i}$, and the extrinsic twist $\omega_{a}^{i j}$, when the appropriate integrability conditions are satisfied. The induced metric is defined by

$$
\gamma_{a b}=e^{\mu}{ }_{a} e_{b}^{\nu} \eta_{\mu \nu} .
$$


This metric, together with its inverse $\gamma^{a b}$, will be used to lower and raise worldvolume indices. The quantity $K_{a b}{ }^{i}=K_{b a}{ }^{i}$ is the extrinsic curvature along the $i$-th normal vector field $n^{\mu}{ }_{i}$,

$$
K_{a b}{ }^{i}=-n_{\mu}{ }^{i} \partial_{a} e^{\mu}{ }_{b}
$$

The extrinsic twist $\omega_{a}^{i j}$, defined by

$$
\omega_{a}^{i j}=\eta_{\mu \nu} n^{\mu j} \partial_{a} n^{\nu i}
$$

is the connection associated with covariance under normal rotations.

To evaluate the variation of these quantities under a worldvolume deformation, we will exploit the covariant formalism describing deformations of the worldvolume geometry developed in Ref. [19]. In this approach, the effect of the deformation on geometrical tensors is covariant not only with respect to reparameterizations of the worldvolume, but also with respect to local rotations of the normals $n^{\mu i}$.

We consider a local action, depending on the embedding functions $Y^{\mu}$, which is both invariant under worldvolume reparametrization, and under rotations of the normals,

$$
S[Y]=\int_{m} \sqrt{-\gamma} L
$$

(For convenience of notation, we have absorbed the worldvolume differential $d^{D} \xi$ into the integral sign. We will do likewise for the integrals over the boundary $\partial m$. ) The lagrangian $L$ is constructed locally from the geometry of the worldvolume $m$

$$
L=L\left(\gamma_{a b}, K_{a b}^{i}, \widetilde{\nabla}_{a} K_{b c}^{i}, \cdots\right),
$$

where we denote by $\widetilde{\nabla}_{a}$ the covariant derivative under rotation of the normal vector fields introduced in 33. For an arbitrary normal vector $\Psi^{i}$, it is defined by

$$
\widetilde{\nabla}_{a} \Psi^{i}=\nabla_{a} \Psi^{i}-\omega_{a}{ }^{i} \Psi^{j}
$$

where $\nabla_{a}$ denotes the (torsionless) worldvolume covariant derivative compatible with $\gamma_{a b}$.

The infinitesimal variation of the action which is induced by a worldvolume deformation can always be decomposed into its tangential and normal parts,

$$
\delta S=\delta_{\|} S+\delta_{\perp} S .
$$

Away from the boundary, the tangential deformation can be identified with a diffeomorphism of $m$, since $\delta_{\|} S$ is a boundary term. Let us examine explicitly how this occurs. We note that $\delta_{\|} f=\Phi^{a} \partial_{a} f$ for any scalar function $f(\xi)$ defined on the worldvolume, $m$. In addition, under a tangential deformation, the induced metric on $m$ tranforms as a Lie derivative,

$$
\delta_{\|} \gamma_{a b}=\nabla_{a} \Phi_{b}+\nabla_{b} \Phi_{a}
$$

Thus

$$
\delta_{\|} \sqrt{-\gamma}=\sqrt{-\gamma} \nabla_{a} \Phi^{a}
$$


For an arbitrary variation of the action, we have

$$
\delta S=\int_{m}\{(\delta \sqrt{-\gamma}) L+\sqrt{-\gamma}(\delta L)\}
$$

A tangential deformation of the worldvolume thus always results in a pure divergence,

$$
\delta_{\|} S=\int_{m} \sqrt{-\gamma} \nabla_{a}\left(L \Phi^{a}\right)=\int_{\partial m} \sqrt{h} L \eta_{a} \Phi^{a},
$$

where we have used Stokes theorem in the second equality, $h$ is the determinant of the metric $h_{A B}$ induced on $\partial m$ by the embeddings described by Eq. (丯),

$$
h_{A B}=\epsilon^{\mu}{ }_{A}{ }^{\nu}{ }_{B} \eta_{\mu \nu},
$$

and $\eta^{a}$ is the unit timelike normal on $\partial m$ pointing into $m$, i.e. the N-velocity of an observer sitting on the hypersurface $\Sigma$. A diffeomorphism of $m$ can only move its boundary.

The action is stationary with respect to tangential deformations of the worldvolume with a vanishing normal component, $\eta_{a} \Phi^{a}=0$ on $\Sigma_{(i)}$ and $\Sigma_{(f)}$. The remaining components of $\Phi^{a}$ may range freely. These are precisely the components that generate a diffeomorphism of the spacelike configurations: the initial and final configurations are fixed, but not the coordinates chosen to describe these configurations.

Whereas the tangential variation of the action is simple, the normal variation is, in general, non-trivial. The normal deformation can always be cast in the form

$$
\delta_{\perp} S=\int_{m} \sqrt{-\gamma}\left[\mathcal{E}_{i}(L) \Phi^{i}+\nabla_{a} \Pi_{i}^{a}\left[\Phi^{i}\right]\right]
$$

i.e. as a worldvolume part, and a pure divergence. Here $\mathcal{E}_{i}(L)$ is the Euler-Lagrange derivative of $L$ projected onto the normals $n^{\mu}{ }_{i}$ to the worldvolume; $\Pi^{a}{ }_{i}$ is a linear differential operator defined on $m$ which arises when the worldvolume gradients of the normal deformation in the worldvolume bulk are confined to a pure divergence, using integration by parts. The argument of the operator $\Pi^{a}{ }_{i}$ is indicated within the square bracket. We can use the divergence theorem in the second term, to obtain,

$$
\delta_{\perp} S=\int_{m} \sqrt{-\gamma} \mathcal{E}_{i}(L) \Phi^{i}+\int_{\partial m} \sqrt{h} \eta_{a} \Pi_{i}^{a}\left[\Phi^{i}\right]
$$

When the classical equations of motion are satisfied,

$$
\mathcal{E}_{i}(L)=0
$$

the action is stationary with respect to normal deformations of $m$. A well posed variational problem requires the vanishing of the boundary terms on the initial and final configurations. The variational principle restricted to normal deformations gives the classical dynamics.

So far, we have considered arbitrary deformations of the embedding functions. Let us now specialize to an infinitesimal Poincaré transformation,

$$
\delta Y^{\mu}=\epsilon^{\mu}+\omega^{\mu}{ }_{\nu} Y^{\nu}
$$


where $\epsilon^{\mu}$ is an infinitesimal constant translation, and an infinitesimal Lorentz transformation is given by $\Lambda^{\mu}{ }_{\nu}=\delta_{\nu}^{\mu}+\omega^{\mu}{ }_{\nu}$, with $\omega_{\mu \nu}=-\omega_{\nu \mu}$. We decompose $\delta Y^{\mu}$ according to Eq.(8). For an infinitesimal spacetime translation $\delta Y^{\mu}=\epsilon^{\mu}$, we have

$$
\Phi_{i}=n^{\mu}{ }_{i} \epsilon_{\mu}, \quad \Phi_{a}=e^{\mu}{ }_{a} \epsilon_{\mu} .
$$

Substituting this into Eqs.(19) and (21), and summing, the variation of the action associated with a spacetime translation can be cast in the form,

$$
\delta S=\epsilon_{\mu} \int_{m}\left[\sqrt{-\gamma} \mathcal{E}^{i}(L) n_{i}^{\mu}+\nabla_{a} \mathcal{P}^{a \mu}\right]
$$

The worldvolume vector density of weight one $\mathcal{P}^{a \mu}$ is given by

$$
\mathcal{P}^{a \mu}=\sqrt{-\gamma}\left(\Pi^{a}{ }_{i}\left[n^{\mu i}\right]+L e^{\mu a}\right) .
$$

This expression for the variation of the action plays a central role in what follows. While tangential deformations do not participate in the variational derivation of the equations of motion, we see that they do contribute in an essential way to the construction of conserved quantities.

The total boundary contribution associated with a translation, using the divergence theorem, is

$$
\delta S=\epsilon_{\mu} \int_{\partial m} \eta_{a} \mathcal{P}^{a \mu}
$$

with the understanding that $\mathcal{P}^{a \mu}$ is to be evaluated at the boundary. This integral is well defined since $\eta_{a} \mathcal{P}^{a \mu}$ is a density of weight one when evaluated there.

Similarly, for an infinitesimal Lorentz transformation, we have

$$
\Phi_{i}=\omega_{\mu \nu} n^{\mu}{ }_{i} Y^{\nu}, \quad \Phi_{a}=\omega_{\mu \nu} e^{\mu}{ }_{a} Y^{\nu}
$$

and in this case the variation of the action associated with a Lorentz transformation reduces to

$$
\delta S=\omega_{\mu \nu} \int_{m}\left[\sqrt{-\gamma} \mathcal{E}^{i}(L) n^{\mu}{ }_{i} Y^{\nu}+\nabla_{a} \mathcal{M}^{a \mu \nu}\right],
$$

where the worldvolume vector density of weight one $\mathcal{M}^{a \mu \nu}$ is given by

$$
\mathcal{M}^{a \mu \nu}=\frac{1}{2} \sqrt{-\gamma}\left[\Pi_{i}^{a}{ }_{i}\left[n^{\mu i} Y^{\nu}\right]+L e^{\mu a} Y^{\nu}-(\mu \leftrightarrow \nu)\right]
$$

The boundary contribution to $\delta S$ gives,

$$
\delta S=\omega_{\mu \nu} \int_{\partial m} \eta_{a} \mathcal{M}^{a \mu \nu}
$$

In our derivation of the expressions for $\mathcal{P}^{a \mu}$ and $\mathcal{M}^{a \mu \nu}$ it is important to emphasize that we did not enforce any boundary conditions on the induced variations. Indeed, it 
would be an error to attempt to enforce the boundary conditions which are appropriate for the variational derivation of the equations of motion.

Let us now suppose that the equations of motion $\mathcal{E}_{i}(L)=0$ are satisfied. We have

$$
\delta S=\epsilon_{\mu}\left[P^{\mu}\left(\Sigma_{(f)}\right)-P^{\mu}\left(\Sigma_{(i)}\right)\right]+\omega_{\mu \nu}\left[M^{\mu \nu}\left(\Sigma_{(f)}\right)-M^{\mu \nu}\left(\Sigma_{(i)}\right)\right] .
$$

The linear momentum $P^{\mu}(\Sigma)$ of the spatial hypersurfaces is defined by

$$
P^{\mu}(\Sigma)=\int_{\Sigma} \eta_{a} \mathcal{P}^{a \mu}
$$

We identify therefore $\mathcal{P}^{a \mu}$ as the linear momentum density. From Eq. (31) on we depart from our earlier convention, understanding the unit normal $\eta^{a}$ to be future pointing when referring to a spacelike hypersurface on $m$.

We emphasize that $P^{\mu}(\Sigma)$ is a quantity associated with the spacelike hypersurface $\Sigma$. In fact, it is possible to express it purely in terms of the geometry of $\Sigma$ itself. We refrain from showing it explicitly, since the investment in additional formalism is not compensated by a corresponding gain in information. On the other hand, the density $\mathcal{P}^{a \mu}$ leads a double life: depending on circumstances, it lives either on the worldvolume $m$, or on its boundary $\partial m$, as exemplified by Eqs. (25) and (27).

We define the angular momentum $M^{\mu \nu}(\Sigma)$ by

$$
M^{\mu \nu}(\Sigma)=\int_{\Sigma} \eta_{a} \mathcal{M}^{a \mu \nu}
$$

which identifies $\mathcal{M}^{a \mu \nu}$ as the angular momentum density.

It is useful to express $\mathcal{M}^{a \mu \nu}$ in the alternative form

$$
\mathcal{M}^{a \mu \nu}=\frac{1}{2}\left[\mathcal{P}^{a \mu} X^{\nu}+\pi^{a}{ }_{i}\left[n^{\mu i} Y^{\nu}\right]-(\mu \leftrightarrow \nu)\right]
$$

where we introduce

$$
\pi^{a}{ }_{i}\left[n^{\mu i} Y^{\nu}\right]=\sqrt{-\gamma}\left(\Pi^{a}{ }_{i}\left[n^{\mu i} Y^{\nu}\right]-n^{\mu i} \Pi^{a}{ }_{i}\left[X^{\nu}\right]\right) .
$$

The antisymmetric part of $\pi^{a}{ }_{i}\left[n^{i \mu} X^{\nu}\right]$ denotes that part of $\mathcal{M}^{a \mu \nu}$ which is not determined completely by the linear momentum density, $\mathcal{P}^{a \mu}$. As we show below, this is precisely the part that is interesting in higher derivative theories.

If the action is Poincaré invariant, so that $\delta S=0$, we have

$$
P^{\mu}\left(\Sigma_{(f)}\right)=P^{\mu}\left(\Sigma_{(i)}\right)
$$

and

$$
M^{\mu \nu}\left(\Sigma_{(f)}\right)=M^{\mu \nu}\left(\Sigma_{(i)}\right) .
$$

However, $\Sigma_{(i)}$ and $\Sigma_{(f)}$ are arbitrarily chosen initial and final configurations of the brane. Thus $P^{\mu}(\Sigma)$ and $M^{\mu \nu}(\Sigma)$ are both independent of the spacelike hypersurface $\Sigma$. In this extremely broad sense, both $P^{\mu}$ and $M^{\mu \nu}$ are constants of the motion.

Our treatment so far has been entirely general. We have not indicated how to evaluate either the Euler-Lagrange derivatives of $L$, or the differential operator, $\Pi^{a}{ }_{i}$. We will consider some concrete examples, beginning with the most elementary, in Sect. 4. 


\section{Equations of motion from momentum conservation}

Before we consider explicit examples, in this section we show how the equations of motion can be expressed in terms of the conservation of the linear momentum.

From the variation of the action under a spacetime translation, Eq. (25), when the action is invariant under translations, so that $\delta S=0$ on the left hand side, we have

$$
\sqrt{-\gamma} \mathcal{E}^{i}(L) n^{\mu}{ }_{i}=-\nabla_{a} \mathcal{P}^{a \mu}
$$

This says that $\nabla_{a} \mathcal{P}^{a \mu}$ is normal to the worldvolume, and the equations of motion imply the conservation of the linear momentum density,

$$
\nabla_{a} \mathcal{P}^{a \mu}=0
$$

and vice versa. That the equations of motions can be restated in terms of the conservation of linear momenta should not come as a surprise. In fact, it is a special case of the fact that the equations of motion can be expressed in terms of the conservation of the stress-energy tensor (for a relativistic string, see e.g. [32]). As we mentioned in the introduction, this is the approach adopted by Carter in his treatment of brane dynamics (see e.g. Ref. [18], and references therein.).

However, the form (37) is not the most useful expression of the conservation law, as it involves the mixed spacetime-worldvolume density, $\mathcal{P}^{a \mu}$; it does not isolate its non-trivial part. It is possible, however, to express the equations of motion in purely worldvolume terms. First, we decompose the spacetime vector density $\mathcal{P}^{a \mu}$ into its tangential and normal parts,

$$
\mathcal{P}^{a \mu}=\mathcal{P}^{a b} e^{\mu}{ }_{b}+\mathcal{P}^{a i} n^{\mu}{ }_{i} .
$$

Note that, in general, the worldvolume tensor field density $\mathcal{P}^{a b}$ will not be symmetric in its indices.

The worldvolume covariant divergence of $\mathcal{P}^{a \mu}$ gives

$$
\nabla_{a} \mathcal{P}^{a \mu}=\left(\nabla_{a} \mathcal{P}^{a b}+K^{b}{ }_{a i} \mathcal{P}^{a i}\right) e^{\mu}{ }_{b}+\left(\widetilde{\nabla}_{a} \mathcal{P}^{a i}-K_{a b}{ }^{i} \mathcal{P}^{a b}\right) n^{\mu}{ }_{i}
$$

where we have made use of the Gauss-Weingarten equations for the worldvolume $m$, (see e.g. [19]),

$$
\begin{aligned}
\nabla_{a} e^{\mu}{ }_{b} & =-K_{a b}{ }^{i} n^{\mu}{ }_{i}, \\
\widetilde{\nabla}_{a} n^{\mu}{ }_{i} & =K_{a b i} e^{\mu b} .
\end{aligned}
$$

The worldvolume projections of the expression (36), using Eq. (39), are therefore given by

$$
\begin{aligned}
\widetilde{\nabla}_{a} \mathcal{P}^{a i}-K_{a b}{ }^{i} \mathcal{P}^{a b} & =-\sqrt{-\gamma} \mathcal{E}^{i}(L) . \\
\nabla_{a} \mathcal{P}^{a b}+K^{b}{ }_{a i} \mathcal{P}^{a i} & =0,
\end{aligned}
$$


The first equation expresses the Euler-Lagrange derivative in a divergence form, so that the equations of motion take the form

$$
\widetilde{\nabla}_{a} \mathcal{P}^{a i}-K_{a b}{ }^{i} \mathcal{P}^{a b}=0
$$

This equation is like a Gauss law for a $O(N-D)$ Yang-Mills "electric field" $\mathcal{P}^{a i}$, with "source" $K_{a b}{ }^{i} \mathcal{P}^{a b}$. Note that only the symmetric part of $\mathcal{P}^{a b}$ enters the equations of motion.

The Bianchi identity (43) is a non-obvious integrability condition required for the existence of solutions of (42). Here, also the antisymmetric part of $\mathcal{P}^{a b}$ contributes.

We will exploit these expressions to examine the different role played by the tangential and normal parts of the momentum density. Moreover, as shown below in Sect. 7, they allow for a novel approach to the linearization of the equations of motion.

We mentioned earlier that $\mathcal{P}^{a b}$ need not be symmetric. However, the conservation of angular momentum, $\nabla_{a} \mathcal{M}^{a \mu \nu}=0$, requires the anti-symmetric part to vanish.

To conclude this section, let us point out that, in the variational principle, one usually keeps volume terms; boundary terms, which contribute to the momenta, are thrown away. The approach via conservation of momenta to the equations of motion points to a complementary strategy: keep only boundary terms, since they also are sufficient to reconstruct the dynamics.

\section{Dirac-Nambu-Goto action}

In order to illustrate the general formalism developed in the previous sections, let us begin with the familiar case of a DNG brane. The DNG action for a relativistic extended object is

$$
S_{(0)}=-\mu \int_{m} \sqrt{-\gamma}
$$

where the constant $\mu$ is the brane tension. This is the simplest action one can write down for such an object. It depends only on the intrinsic geometry of the worldvolume.

The normal deformation of this action is given by

$$
\delta_{\perp} S_{(0)}=-\mu \int_{m} \sqrt{-\gamma} K_{i} \Phi^{i}
$$

where we use the familiar expression relating the Lie derivative along the normals of the volume element of $m$ to its mean extrinsic curvature $K^{i}=\gamma^{a b} K_{a b}{ }^{i}$,

$$
\delta_{\perp} \sqrt{-\gamma}=\sqrt{-\gamma} K^{i} \Phi_{i}
$$

The tangential deformation of this action is simply given by Eq. (19), with $L=-\mu$.

In this geometrical language, the equations of motion are given by the vanishing of the mean extrinsic curvature,

$$
-\mu K^{i}=0
$$


The worldvolumes that extremize the DNG action are extremal timelike surfaces. This is a system of $N-D$ second-order hyperbolic partial differential equations for the embedding functions, $Y^{\mu}(\xi)$. The appropriate boundary conditions in the variational principle are $\eta_{a} \Phi^{a}=0$ on $\partial m$. Both $\Phi^{i}$ and $\Phi_{\|}^{a}=\Phi^{a}-\eta_{b} \Phi^{b} \eta^{a}$ are arbitrary. We only need to fix the initial and final hypersurface geometries. Anything more is superfluous.

To bring the equations of motion into a more familiar form, using the Gauss-Weingarten equations (40), we have that

$$
K^{i}=-n_{\mu}^{i} \Delta Y^{\mu}
$$

where $\Delta$ is the worldvolume d'Alembert operator. The tangential projections of $\Delta Y^{\mu}$ vanish identically. We can now peel Eq.(49), and its tangential counterpart to recover the familiar harmonicity condition,

$$
\mu \Delta Y^{\mu}=0 .
$$

Note that in this model there is no surface term arising from the normal variation, so that we have that the operator introduced in Eq. (21) vanishes identically, $\Pi^{a}{ }_{i}\left[\Phi^{i}\right]=0$. This is a feature which is unique to the DNG action.

The invariance of the DNG action under Poincaré transformations gives the linear momentum density

$$
\mathcal{P}^{a \mu}=-\mu \sqrt{-\gamma} e^{\mu a},
$$

so that the total momentum $P^{\mu}(\Sigma)$ is given by

$$
P^{\mu}(\Sigma)=-\mu \int_{\Sigma} \sqrt{h} \eta^{\mu}
$$

where $\eta^{\mu}=\eta^{a} e^{\mu}{ }_{a}$ is the unit velocity vector at a given point on $\Sigma$. The momentum density $\mathcal{P}^{a \mu}$ is not only tangent to the worldsheet, it also lies parallel to the tangent vector, $e^{\mu}{ }_{a}$. In this sense, extremal surfaces, like geodesics, are self-parallel.

The linear momentum of a DNG brane is defined directly in terms of initial data on the spacelike hypersurface $\Sigma$, without explicit reference to the worldvolume that will be generated by these initial data. This expression for the linear momentum of a DNG brane generalizes the expression for a free relativistic massive particle, $P^{\mu}=m U^{\mu}$, with $m$ its mass, and $U^{\mu}$ its unit velocity.

The worldvolume projections of $\mathcal{P}^{a \mu}$ are, respectively,

$$
\begin{aligned}
& \mathcal{P}^{a b}=-\mu \sqrt{-\gamma} \gamma^{a b}, \\
& \mathcal{P}^{a i}=0 .
\end{aligned}
$$

The vanishing of the normal part was to be expected - the DNG equations of motion are of second order in the embedding functions. Moreover, the tangential projection is explicitly symmetric. Substituting these projections into the worldvolume projections of the linear momentum conservation equation, Eqs. (42) and (43), we find that the first reproduces the extremal dynamics, $-\mu K^{i}=0$, whereas the second is satisfied identically. We also note that $\mathcal{P}^{a b}$ is scale invariant, under $\gamma_{a b} \rightarrow \Omega^{2} \gamma_{a b}$, if and only if $D=2$, i.e. for a 
relativistic string. This is a consequence of the scale invariance of the DNG string, which becomes manifest in the Polyakov formulation of the theory [4].

The angular momentum density is simply

$$
\mathcal{M}^{a \mu \nu}=\mathcal{P}^{a[\mu} X^{\nu]}
$$

When the equations of motion hold, this is automatically conserved. The total angular momentum $M^{\mu \nu}(\Sigma)$ is given by

$$
M^{\mu \nu}(\Sigma)=\frac{\mu}{2} \int_{\Sigma} \sqrt{h}\left\{\eta^{\mu} X^{\nu}-(\mu \leftrightarrow \nu)\right\}
$$

Having covered the familiar case of a DNG brane, in the next section, we move on to a less trivial class of applications.

\section{$5 \quad$ Extrinsic curvature actions}

In this section, we consider the simplest actions involving extrinsic curvature, described by some lagrangian $L=L\left(\gamma^{a b}, K_{a b}{ }^{i}\right)$. The more general case of a lagrangian that depends on derivatives of the extrinsic curvature as well can be treated along the same lines, and we will consider it in an Appendix. The possibilities are limited by the requirement that the lagrangian must transform as a scalar under diffeomorphisms of the worldvolume, and under rotations of the normals. In general, such theories will involve derivatives of the embedding functions higher than first, since the extrinsic curvature generalizes to a brane the acceleration of a point particle.

For a hypersurface, $D=N-1$, a lagrangian proportional to (odd powers of) $K$ is admissible. For arbitrary co-dimension, however, the lowest order lagrangian invariant under normal rotations is quadratic in $K_{a b}{ }^{i}$,

$$
L=-\mu+\alpha_{1} K^{i} K_{i}+\alpha_{2} K_{a b}{ }^{i} K^{a b}{ }_{i},
$$

where $\alpha_{1}$ and $\alpha_{2}$ are constants that measure the rigidity of the brane.

The worldvolume scalars $K^{i} K_{i}$ and $K_{a b}{ }^{i} K^{a b}{ }_{i}$ are not independent. The completely contracted Gauss-Codazzi equations in a flat spacetime background relates their difference to the worldvolume scalar curvature $\mathcal{R}$, with

$$
\mathcal{R}=K^{i} K_{i}-K^{a b i} K_{a b i} .
$$

When $D=2$, for a (closed) relativistic string, the action constructed from $\mathcal{R}$ is a topological invariant, as follows from the Gauss-Bonnet theorem, so that the actions determined by the two quadratics are locally equivalent.

As before, the tangential variation of the action is straightforward, see Eq. (19). We exploit the chain rule to write down the normal variation of the lagrangian in terms of the variations of its arguments. We then have

$$
\delta_{\perp} S=\int_{m} \sqrt{-\gamma}\left\{K^{i} \Phi_{i} L+L_{i}^{a b}\left(\tilde{\delta}_{\perp} K_{a b}{ }^{i}\right)+L_{a b}\left(\delta_{\perp} \gamma^{a b}\right)\right\}
$$


where we have used Eq. (47) for the first term, and we have defined,

$$
\begin{array}{r}
L_{i}^{a b}=\frac{\partial L}{\partial K_{a b}{ }^{i}}=L_{i}^{b a}, \\
L_{a b}=\frac{\partial L}{\partial \gamma^{a b}}=L_{b a} .
\end{array}
$$

We now exploit the results of Ref. [19], specialized to a flat background, to express the normal variation of the inverse induced metric and the extrinsic curvature as,

$$
\begin{aligned}
\delta_{\perp} \gamma^{a b} & =-2 K^{a b}{ }_{i} \Phi^{i} \\
\tilde{\delta}_{\perp} K_{a b}{ }^{i} & =-\widetilde{\nabla}_{a} \widetilde{\nabla}_{b} \Phi^{i}+K_{a c}{ }^{i} K_{b j}^{c} \Phi^{j} .
\end{aligned}
$$

The deformation operator $\tilde{\delta}_{\perp}$ acting on $K_{a b}{ }^{i}$ is constructed analogously to $\widetilde{\nabla}_{a}$, and it involves a deformation connection $\gamma_{i j}=-\gamma_{j i}$, so that when acting e.g. on a vector under normal rotations, it is defined by

$$
\tilde{\delta}_{\perp} A^{i}=\delta_{\perp} A^{i}-\gamma_{i}^{j} A_{j}
$$

This refinement is necessary to ensure covariance of the deformation under normal rotations, but coinciding with $\delta_{\perp}$ when considering the deformation of normal rotation scalars. (For more detail the reader may refer to $[19$. .)

We remove the hessian of $\Phi^{i}$ appearing in the second term on the right hand side of Eq.(59), when we insert Eq. (63). The result is

$$
\begin{aligned}
\delta_{\perp} S & =\int_{m} \sqrt{-\gamma}\left\{K^{i} L-2 K^{a b i} L_{a b}-\widetilde{\nabla}_{a} \widetilde{\nabla}_{b} L^{a b}{ }_{i}-K_{a c i} K_{b}{ }^{c j} L^{a b}{ }_{j}\right\} \Phi^{i} \\
& +\int_{m} \sqrt{-\gamma} \nabla_{a}\left\{-L^{a b}{ }_{i} \widetilde{\nabla}_{b} \Phi^{i}+\left(\widetilde{\nabla}_{b} L^{a b}{ }_{i}\right) \Phi^{i}\right\} .
\end{aligned}
$$

We identify the Euler-Lagrange derivative as

$$
\mathcal{E}_{i}(L)=-\widetilde{\nabla}_{a} \widetilde{\nabla}_{b} L^{a b}{ }_{i}+L^{a b}{ }_{j} K_{a c}{ }^{j} K^{c}{ }_{b i}+L K_{i}-2 L_{a b} K^{a b}{ }_{i} .
$$

Generically, the Euler-Lagrange equations $\mathcal{E}^{i}=0$ are of second order in derivatives of $K_{a b}{ }^{i}$, so they are of fourth order in derivatives of the embedding functions $Y^{\mu}$.

Appropriate boundary conditions in the variational principle are that $\Phi^{i}=0$, in order to cancel the last term in Eq. (65), and, since this implies already the vanishing of the derivative of $\Phi^{i}$ along $\Sigma$, in order to cancel the next to last term in Eq. (65), we need only to require $\eta^{a} \widetilde{\nabla}_{a} \Phi^{i}=0$ on $\partial m$, independently of $L^{a b}{ }_{i}$.

We identify the operator $\Pi^{a}{ }_{i}$ introduced in Eq.(21) as the covariant 'wronskian':

$$
\Pi^{a}{ }_{i}\left[\Phi^{i}\right]=-L^{a b}{ }_{i} \widetilde{\nabla}_{b} \Phi^{i}+\left(\widetilde{\nabla}_{b} L^{a b}{ }_{i}\right) \Phi^{i}
$$

In particular, for a $\Phi^{i}$ which corresponds to a background translation,

$$
\epsilon_{\mu} \Pi^{a}{ }_{i}\left[n^{\mu i}\right]=\epsilon_{\mu}\left[-L^{a b}{ }_{i} K_{b}{ }^{c i} e^{\mu}{ }_{c}+\left(\widetilde{\nabla}_{b} L^{a b}{ }_{i}\right) n^{\mu i}\right] .
$$


where we exploit the Gauss-Weingarten equation (41) to simplify the first term. We thus have from Eq. (26), the general expression for the linear momentum density,

$$
\mathcal{P}^{a \mu}=\sqrt{-\gamma}\left[\left(L \gamma^{a b}-L_{i}^{a c} K^{b}{ }_{c i}\right) e^{\mu}{ }_{b}+\left(\widetilde{\nabla}_{b} L^{a b}{ }_{i}\right) n^{\mu i}\right] .
$$

Unlike the DNG case, in general, the momentum density $\mathcal{P}^{a \mu}$ now possesses a component normal to the worldvolume.

We can write the tangential part in a different way. First we separate the quantity $L_{i}^{a c} K^{b}{ }_{c i}$ in its symmetric and anti-symmetric parts. It is easy to show that the symmetric part is given by

$$
L^{c a}{ }_{i} K^{b}{ }_{c}^{i}+L^{c b}{ }_{i} K^{a}{ }_{c}^{i}=2 L^{a b} .
$$

Then, we can rewrite the tangential part of $\mathcal{P}^{a \mu}$ in the form

$$
\mathcal{P}^{a b}=\sqrt{-\gamma}\left(L \gamma^{a b}-L^{a b}\right)+\mathcal{Q}^{a b}
$$

where we isolate the anti-symmetric part of $\mathcal{P}^{a b}$,

$$
\mathcal{Q}^{a b}=\sqrt{-\gamma}\left(L^{c a}{ }_{i} K^{b}{ }_{c i}-L^{c b}{ }_{i} K^{a}{ }_{c i}\right)=0,
$$

which vanishes identically for the geometrical actions we consider.

In order to check in a non-trivial case that the equations of motion can be expressed in the form (44), we can substitute the normal and tangential projections into the left hand side of Eq.(42), we obtain the equations of motion in the form (66). Moreover, we can check that Eq.(43), corresponding to a worldvolume translation, is in fact an identity.

Inspection of the form (42) of the equations of motion, or directly of Eq. (66), shows that the necessary and sufficient condition for the equations of motion to be of second order in derivatives of the embedding functions is simply that the normal component of the linear momentum density vanishes, $\mathcal{P}^{a i}=0$.

The alternative expression (171) for the tangential part of the linear momentum density is also useful for recovering the result that invariance of the action under scale transformations implies

$$
\mathcal{P}^{a b} \gamma_{a b}=0
$$

To see this, consider that, using Eq. (18), the variation of the action under a change of the worldvolume metric is simply

$$
\begin{aligned}
\delta S & =\int_{m} \sqrt{-\gamma}\left[L \gamma^{a b}-L^{a b}\right] \delta \gamma_{a b} \\
& =\int_{m} \mathcal{P}^{a b} \delta \gamma_{a b},
\end{aligned}
$$

The second line follows from the fact that only the symmetric part of $\mathcal{P}^{a b}$ enters. This identifies the symmetric part of $\mathcal{P}^{a b}$ as the worldvolume stress-energy tensor. When the induced metric undergoes a scale transformation, scale invariance of the action, $\delta S=0$, implies the tracelessness condition $(\overline{73})$, and viceversa. 
Let us now move on to the angular momentum. The operator appearing in Eq.(35) is

$$
\pi^{a}{ }_{i}\left[n^{i \mu} X^{\nu}\right]=-L^{a b}{ }_{i} n^{\mu i} e^{\nu}{ }_{b},
$$

so that the angular momentum density is given by

$$
\mathcal{M}^{a \mu \nu}=\frac{1}{2}\left[\mathcal{P}^{a \mu} X^{\nu}-\sqrt{-\gamma} L^{a b}{ }_{i} n^{\mu i} e^{\nu}{ }_{b}-(\mu \leftrightarrow \nu)\right] .
$$

which does not involve derivatives in $K_{a b}{ }^{i}$ other than those already contained in $\mathcal{P}^{a \mu}$. The second term may be thought of as an effect of the finite width of the worldvolume, when we go beyond the DNG approximation. Its appearance is necessary to ensure conservation of angular momentum. Neither term alone is conserved. To see this, let us assume that the equations of motion hold or, equivalently, that the linear momentum is conserved, $\nabla_{a} \mathcal{P}^{a \mu}=0$. The divergence of the angular momentum density is

$$
\begin{aligned}
\nabla_{a} \mathcal{M}^{a \mu \nu}=\frac{1}{2}\left[\mathcal{P}^{a \mu} e^{\nu}{ }_{a}\right. & -\sqrt{-\gamma}\left(\widetilde{\nabla}_{a} L^{a b}{ }_{i}\right) n^{\mu i} e^{\nu}{ }_{b}-\sqrt{-\gamma} L^{a b}{ }_{i} K_{a}{ }^{c i} e^{\mu}{ }_{c} e^{\nu}{ }_{b} \\
& \left.+\sqrt{-\gamma} L^{a b}{ }_{i} K_{a b j} n^{\mu i} n^{\nu j}-(\mu \leftrightarrow \nu)\right],
\end{aligned}
$$

where we have used the Gauss-Weingarten equations (40), (41). In this expression, all of the possible spacetime bivectors appear. However, if we express $\mathcal{P}^{a \mu}$ in terms of its projections, we obtain

$$
\left.\nabla_{a} \mathcal{M}^{a \mu \nu}=\frac{1}{2}\left[2 \mathcal{Q}^{a b} e^{\mu}{ }_{a} e^{\nu}{ }_{b}-\sqrt{-\gamma} L^{a b}{ }_{i} K_{a b j} n^{\mu i} n^{\nu j}-(\mu \leftrightarrow \nu)\right)\right] .
$$

The two terms are independent, the first is proportional to a bivector parallel to the worlvolume, the second to a bivector normal to it. Therefore the conditions necessary for conservation of angular momentum are

$$
\begin{aligned}
\mathcal{Q}^{a b} & =0, \\
L^{a b}{ }_{i} K_{a b j}-L^{a b}{ }_{j} K_{a b i} & =0 .
\end{aligned}
$$

The first condition was to be expected from classical elasticity theory [34, and, as mentioned above, is satisfied identically. The second condition is a new "thickness" effect associated with co-dimension $N-D>1$, and it is also satisfied identically for the geometrical actions we consider.

We also note that at this order, $\mathcal{M}^{a \mu \nu}$ does not involve any term proportional to $n^{\mu i} n^{\nu j}-(\mu \leftrightarrow \nu)$, which is permitted if $N-D>1$. Such a term will show up in higher order theories, as we show in Appendix A.

\section{Extrinsic curvature actions: examples}

In order to make our discussion more concrete, in this section we consider some specific examples of the class of theories treated in the previous section. We begin with the simple 
case of a hypersurface action of the form

$$
S_{(1)}=\alpha_{0} \int_{m} \sqrt{\gamma} K
$$

Since $L_{a b}=\alpha_{0} K_{a b}$ and $L^{a b}{ }_{\perp}=\alpha_{0} \gamma^{a b}$ (we use the symbol $\perp$ to denote the only normal direction), from Eq. (66), we find the Euler-Lagrange derivative in the form

$$
\mathcal{E}_{\perp}=\alpha_{0}\left(K^{2}-K^{a b} K_{a b}\right)=\alpha_{0} \mathcal{R},
$$

where we have used the contracted Gauss-Codazzi equation (58). This action is extremized by worldvolumes with vanishing scalar Ricci curvature. It is a topological invariant (the winding number) for a pointlike trajectory. The equations of motion, like the DNG case, are of second order in time derivatives of the embedding functions. Indeed, the total linear momentum is given by

$$
P^{\mu}(\Sigma)=\alpha_{0} \int_{\Sigma} \sqrt{h} \eta_{a}\left(K \gamma^{a b}-K^{a b}\right) e_{b}^{\mu}
$$

and we see that the linear momentum density is purely tangential, and manifestly symmetric. Moreover, reading off the quantity $\mathcal{P}^{a b}$, and substituting in the equations of motion in the form (43), reproduces $\mathcal{E}_{\perp}=0$, with the Euler-Lagrange derivative given by Eq. (82).

Although the addition of this action to the DNG action does not change the order of the equations of motion, it does change the boundary conditions. Now we need to require $\eta^{a} \widetilde{\nabla}_{a} \Phi^{i}=0$. We will discuss this issue in more detail below, in the special case of a Einstein-Hilbert brane action.

The total angular momentum is

$$
M^{\mu \nu}(\Sigma)=\frac{1}{2} \int_{\Sigma} \eta_{a}\left[\mathcal{P}^{a \mu} X^{\nu}-\alpha_{0} \sqrt{h} n^{\mu} e^{\nu a}-(\mu \leftrightarrow \nu)\right]
$$

The second term is proportional to the normal bivector $n^{\mu} \eta^{\nu}-n^{\nu} \eta^{\mu}$. In the equation for angular momentum conservation, $\nabla_{a} \mathcal{M}^{a \mu \nu}=0$, both terms are conserved separately. The first because of the vanishing of $\mathcal{P}^{a i}$ and the symmetry of $\mathcal{P}^{a b}$. The second is conserved automatically, because the condition (80) is vacuus. Alternatively, this can be checked directly using the Gauss-Weingarten equations (40), (41), specialized to the case of a hypersurface.

Let us now examine the case of an action quadratic in the extrinsic curvature. In particular, let us consider first,

$$
S_{(2, a)}=\alpha_{1} \int_{m} \sqrt{-\gamma} K^{i} K_{i}
$$

We have $L_{a b}=2 \alpha_{1} K^{i} K_{a b i}$, and $L^{a b}{ }_{i}=2 \alpha_{1} K_{i} \gamma^{a b}$. Thus from Eq. (66), the Euler -Lagrangian derivative is

$$
\mathcal{E}_{i}=-2 \alpha_{1}\left[\widetilde{\Delta} K_{i}+K^{a b}{ }_{i} K_{a b}{ }^{j} K_{j}-\frac{1}{2} K^{j} K_{j} K_{i}\right] .
$$


For a quadratic action, the Euler-Lagrange derivative is proportional to $\tilde{\Delta} K_{i}$ plus some cubic in $K_{a b}^{i}$. We note that the extremal solutions $K^{i}=0$ continue to be solutions of this particular theory 35.

The linear momentum density takes the form

$$
\mathcal{P}^{a \mu}=\alpha_{1} \sqrt{-\gamma}\left\{K_{i}\left(K^{i} \gamma^{a b}-2 K^{a b i}\right) e^{\mu}{ }_{b}+2\left(\widetilde{\nabla}_{a} K_{i}\right) n^{\mu i}\right\}
$$

Thus, on all extremal solutions of the theory defined by $S=S_{(0)}+S_{(2, a)}$, with $K^{i}=0$, the rigidity makes no contribution to the momentum. If the complete action is given by the rigidity term, solutions with $K^{i}=0$ carry no momentum. This was noted by Boisseau and Letelier in 25] for the special case of a relativistic string.

The scale invariance of this action in the case of a relativistic string $(D=2)$ can be checked by verifying that $\mathcal{P}^{a b} \gamma_{a b}=0$.

The normal projection of the linear momentum density is

$$
\mathcal{P}^{a i}=2 \alpha_{1} \sqrt{-\gamma} \widetilde{\nabla}^{a} K^{i}
$$

Therefore, if the mean curvature is constant, $K^{i}=$ const., so that $\mathcal{P}^{a i}=0$, then the equations of motion are of second order in derivatives of the embedding functions.

The angular momentum density is given by

$$
\mathcal{M}^{a \mu \nu}=\frac{1}{2}\left[\mathcal{P}^{a \mu} X^{\nu}-2 \sqrt{-\gamma} K_{i} n^{\mu i} e^{\nu a}-(\mu \leftrightarrow \nu)\right] .
$$

The second term is proportional to the bivector normal to $\Sigma$. Both conditions (79), (80) are identically satisfied, so that angular momentum is conserved.

The total angular momentum $M^{\mu \nu}$ also vanishes when $K^{i}=0$. For a DNG string, the well-known Regge inequality bounds the spin by the mass. We conclude that for a rigid string, the inequality continues to hold for all DNG solutions [35].

We have seen already that for strings the two quadratics are not independent so that the $K^{i} K_{i}$ theory is, in fact, the unique theory quadratic in extrinsic curvature. In general, for arbitrary $D$, however we also have the other possibility,

$$
S_{(2, b)}=\alpha_{2} \int_{m} \sqrt{-\gamma} K_{a b}^{i} K_{i}^{a b} .
$$

We find easily that $L_{a b}=2 \alpha_{2} K^{c}{ }_{a i} K_{b c}{ }^{i}$ and $L^{a b}{ }_{i}=2 \alpha_{2} K^{a b}{ }_{i}$. From Eq. (66), the EulerLagrange derivative is

$$
\mathcal{E}_{i}=-2 \alpha_{2}\left(\widetilde{\nabla}_{a} \widetilde{\nabla}_{b} K^{a b}{ }_{i}+K^{a c}{ }_{j} K_{c b}^{j} K_{a i}{ }^{b}-\frac{1}{2} K_{a b}{ }^{j} K^{a b}{ }_{j} K_{i}\right) .
$$

This expression for the Euler-Lagrange derivative is perfectly legitimate. On the other hand, we can offer an alternative expression, closer to the one given for the Euler-Lagrange derivative of the other quadratic action, Eq. (86). We use the once contracted GaussCodazzi equation,

$$
\mathcal{R}_{a b}-K^{i} K_{a b i}+K_{a c}^{i} K_{b i}^{c}=0
$$


and the contracted Codazzi-Mainardi integrability condition,

$$
\widetilde{\nabla}_{a}\left(K^{a b i}-\gamma^{a b} K^{i}\right)=0,
$$

to reduce the Euler-Lagrange derivative (91) to the form

$$
\mathcal{E}_{i}=-2 \alpha_{1}\left(\widetilde{\Delta} K_{i}+K^{a b}{ }_{i} K_{a b}{ }^{j} K_{j}-\frac{1}{2} K^{j} K_{j} K_{i}-\mathcal{G}_{a b} K^{a b}{ }_{i}\right),
$$

where $\mathcal{G}_{a b}=\mathcal{R}_{a b}-(1 / 2) \mathcal{R} \gamma_{a b}$ is the worldvolume Einstein tensor. This alternative expression is identical to Eq. (86), except for the addition of the last term, which vanishes identically for a string, $D=2$.

The linear momentum density for this theory is

$$
\mathcal{P}^{a \mu}=\alpha_{2} \sqrt{-\gamma}\left[\left(K_{c d}{ }^{i} K^{c d}{ }_{i} \gamma^{a b}-2 K^{a}{ }_{c i} K^{b c i}\right) e^{\mu}{ }_{b}+2\left(\widetilde{\nabla}_{b} K^{a b i}\right) n^{\mu}{ }_{i}\right] .
$$

We can use the Codazzi-Mainardi equation (93) to simplify the second term, and the contractions of the Gauss-Codazzi equation (58), (92), to write the linear momentum density in the alternative form,

$$
\mathcal{P}^{a \mu}=\alpha_{2} \sqrt{-\gamma}\left[\left(K_{i} K^{i} \gamma^{a b}-2 K_{i} K^{a b i}-2 \mathcal{G}^{a b}\right) e^{\mu}{ }_{b}+2\left(\widetilde{\nabla}^{a} K^{i}\right) n^{\mu}{ }_{i}\right] .
$$

We note that also for this theory, for constant mean curvature solutions, $K^{i}=$ const., the equations of motion are of second order in derivatives of the embedding functions. Moreover, for $D=2$, the theory is scale invariant, since $\mathcal{G}_{a b}=0$, identically, so that $\mathcal{P}^{a b} \gamma_{a b}=0$.

The total angular momentum is

$$
M^{\mu \nu}(\Sigma)=\frac{1}{2} \int_{\partial m} \eta_{a}\left[\mathcal{P}^{a \mu} X^{\nu}+2 \alpha_{2} \sqrt{h} K^{a b i} n^{\mu}{ }_{i} e^{\nu}{ }_{b}-(\mu \leftrightarrow \nu)\right] .
$$

Whereas in the cases considered previously the second term, independent of the linear momentum density, involved only the bivector normal to $\Sigma$, here it includes also the bivector normal-tangential to $\Sigma$.

As a last example of an action quadratic in the extrinsic curvature, we consider now the Einstein-Hilbert action,

$$
S_{E H}=\beta \int_{m} \sqrt{-\gamma} \mathcal{R} .
$$

We have,

$$
\begin{aligned}
L_{a b} & =2 \beta\left(K_{i} K_{a b}{ }^{i}-K_{a c i} K_{b}{ }^{c i}\right)=2 \beta \mathcal{R}_{a b}, \\
L^{a b}{ }_{i} & =2 \beta\left(K_{i} \gamma^{a b}-K^{a b}{ }_{i}\right) .
\end{aligned}
$$

Note that the Codazzi-Mainardi equation (93) implies

$$
\widetilde{\nabla}_{b} L^{a b}{ }_{i}=0 .
$$


This kills the boundary term proportional to $\Phi_{i}$.

To calculate the variation of this action, we can use the contracted Gauss-Codazzi equation for a flat space-time background, Eq.(58), and the results of the previous section. We obtain,

$$
\begin{aligned}
\delta S_{E H} & =\beta \int_{m} \sqrt{-\gamma}\left\{-2 K^{a b i} \mathcal{G}_{a b} \Phi_{i}\right\} \\
& +\beta \int_{\partial m} \sqrt{h} \eta_{a}\left\{2\left[K^{a b i}-\gamma^{a b} K^{i}\right] \widetilde{\nabla}_{b} \Phi_{i}+\mathcal{R} \Phi^{a}\right\},
\end{aligned}
$$

where $\mathcal{G}_{a b}$ is the worldvolume Einstein tensor, $\mathcal{G}_{a b}=\mathcal{R}_{a b}-\frac{1}{2} \gamma_{a b} \mathcal{R}$. It follows at once that the Euler-Lagrange derivative of the Einstein-Hilbert action is given by

$$
\mathcal{E}_{i}=-2 \beta K^{a b}{ }_{i} \mathcal{G}_{a b} .
$$

When $D=2$, for a string, the Einstein tensor vanishes identically, $\mathcal{G}_{a b}=0$ and $\mathcal{E}_{i}=0$ identically. This can also be checked by considering the difference of the Euler-Lagrange derivatives for the two actions quadratic in the curvature, Eqs. (86), (94).

In general, a DNG action with an Einstein-Hilbert correction satisfies the Euler-Lagrange equations,

$$
K^{a b}{ }_{i}\left(\mu \gamma_{a b}+2 \beta \mathcal{G}_{a b}\right)=0 .
$$

In particular, any embedded Einstein manifold, satisfying $\mathcal{G}_{a b}=-(\mu / 2 \beta) \gamma_{a b}$ is a solution.

We note that an Einstein-Hilbert addition to the DNG action does not change the order of the equations of motion. They remain second order. The action, however, is no longer stationary under the same boundary conditions as the DNG theory, even when $D=2$. We now need to require that $\eta^{a} \widetilde{\nabla}_{a} \Phi^{i}=0$. Technically, we trace this necessity to the fact that the action contains a term linear in the second derivative of the induced metric. In general, this spells trouble in a functional integral approach to the quantum theory 223. The resolution is, of course, well known: introduce a surface correction to the action to cancel these offending surface terms. The essence of the variational problem is encountered in the elementary description of a free particle by the lagrangian, $L(x, \ddot{x})=-(1 / 2) x \ddot{x}$ which is equivalent to the lagrangian $(1 / 2) \dot{x}^{2}$ with the addition of a boundary term to the corresponding action. This term is simply $(1 / 2) x \dot{x}$. To do this in a covariant way for the problem at hand is a little harder. We are guided by the analogous problem in general relativity. This issue is treated in Sect. 8.

Here we examine the conserved quantitied associated with the unadorned EinsteinHilbert action. With or without surface modifications, Noether's theorem applies so long as the action possesses the required invariance. Noether's theorem is independent of the particular boundary conditions that are invoked to determine the Euler-Lagrange equations.

The Einstein-Hilbert contribution to the momentum density is given by

$$
\begin{aligned}
\mathcal{P}^{a \mu} & =\beta \sqrt{-\gamma}\left[2\left(K_{a b}{ }^{i}-\gamma_{a b} K^{i}\right) K_{i}^{b c}+\mathcal{R}\right] e^{\mu}{ }_{c} \\
& =-2 \beta \sqrt{-\gamma} \mathcal{G}^{a b} e^{\mu}{ }_{b},
\end{aligned}
$$


where we have exploited the once contracted Gauss-Codazzi relation, to obtain the second line. We note that the vanishing of this momentum density gives the vacuum Einstein field equations for the worldvolume. If one is interested in an embedding formulation of GR in a flat background spacetime, this observation isolates the relevant subspace of the space of solutions, solving the problem posed by Regge and Teitelboim in [20]. We plan to develop the consequences of this observation elsewhere.

The angular momentum density $\mathcal{M}^{a \mu \nu}$ decomposes into a sum of two separately conserved quantities:

$$
\mathcal{M}^{a \mu \nu}=\frac{1}{2}\left[\mathcal{P}^{a \mu} X^{\nu}+2 \beta \sqrt{-\gamma}\left(K^{a b i}-\gamma^{a b} K^{i}\right) n^{\mu}{ }_{i} e^{\nu}{ }_{b}-(\mu \leftrightarrow \nu)\right] .
$$

Again, both conditions (79) and (80) are identically satisfied, so the total angular momentum is conserved.

In the case of a string with worldvolume dimension $D=2$, the Einstein-Hilbert lagrangian is a topological invariant and does not contribute to the equations of motion. We would hope that the corresponding contributions to the conserved quantities also vanish. As expected $\mathcal{P}^{a \mu}=0$; however, whereas the first term vanishes in $\mathcal{M}^{a \mu \nu}$, the second does not. However, as we have seen, this non-vanishing part is conserved kinematically, it is divergence free off shell. One is always at liberty to add such a tensor to produce a 'new' conservation law. The addition of the appropriate boundary term, as shown below in Sect. 8 , removes this extra kinematic term.

\section{Linearized equations of motion from momenta}

In this section, we consider the second order variation of the action, and we show how the linearized equations of motion can be expressed in terms of the normal variation of the linear momentum density $\mathcal{P}^{a \mu}$. It turns out that this approach to the linearized equations of motion is more economical than a direct approach, which considers the variation of the Euler-Lagrange derivative, $\delta \mathcal{E}^{i}(L)$ [36, 33, 37, 38, 19]. The virtue of this approach is that it involves the variation of geometrical quantities of lower order in derivatives of the embedding functions.

In Sect. 3, we have shown explicitly how to express the equations of motion in terms of the conservation of the appropriate projections of the momenta. The key equation we used, for an infinitesimal translation, is Eq. (25). We consider only its normal variation for the moment. We obtain

$$
\delta_{\perp}(\delta S)=\epsilon_{\mu}\left\{\int_{m} \sqrt{-\gamma}\left[K^{j} \Phi_{j} \mathcal{E}^{i} n^{\mu}{ }_{i}+\left(\tilde{\delta}_{\perp} \mathcal{E}^{i}\right) n^{\mu}{ }_{i}-\mathcal{E}^{i}\left(\widetilde{\nabla}_{a} \Phi_{i}\right) e^{\mu a}\right]+\int_{m} \nabla_{a} \delta_{\perp} \mathcal{P}^{a \mu}\right\} .
$$

Here we have used Eq. (47), and the second normal deformation Gauss-Weingarten equation [19],

$$
\tilde{\delta}_{\perp} n^{\mu}{ }_{i}=\left(\widetilde{\nabla}_{a} \Phi^{i}\right) e^{\mu a}
$$


In the last term, we have exploited the fact that, since $\mathcal{P}^{a \mu}$ is a vector density of weight one, variation and covariant differentiation commute, $\delta \nabla_{a} \mathcal{P}^{a \mu}=\nabla_{a} \delta \mathcal{P}^{a \mu}$.

When the action is invariant under translations, $\delta S=0$, this equation relates the normal variation of the Euler-Lagrange derivative to the divergence of the normal variation of the linear momentum density.

Following the same strategy we used for the equations of motion, let us decompose $\mathcal{P}^{a \mu}$ into its worldvolume projections,

$$
\delta_{\perp} \mathcal{P}^{a \mu}=\left[\delta_{\perp} \mathcal{P}\right]^{a b} e^{\mu}{ }_{b}+\left[\delta_{\perp} \mathcal{P}\right]^{a i} n^{\mu}{ }_{i},
$$

where we use the brackets notation in order to distinguish the projection of the variation from the variation of the projection, since in general they are different, e.g. $\left[\delta_{\perp} \mathcal{P}\right]^{a b} \neq$ $\delta_{\perp} \mathcal{P}^{a b}$. In fact, this is the reason that makes the approach described here more efficient than a direct approach.

A straightforward calculation gives that when the action is invariant under translations, so that $\delta S=0$ on the left hand side of Eq. (104), the worldvolume projections of Eq. (104) are

$$
\begin{aligned}
\sqrt{-\gamma}\left(\tilde{\delta}_{\perp} \mathcal{E}^{i}\right) & =-\sqrt{-\gamma} K^{j} \Phi_{j} \mathcal{E}^{i}-\widetilde{\nabla}_{a}\left[\delta_{\perp} \mathcal{P}\right]^{a i}+\left[\delta_{\perp} \mathcal{P}\right]^{a b} K_{a b}{ }^{i} \\
\sqrt{-\gamma} \mathcal{E}^{i}\left(\widetilde{\nabla}^{b} \Phi_{i}\right) & =\widetilde{\nabla}_{a}\left[\delta_{\perp} \mathcal{P}\right]^{a b}+\left[\delta_{\perp} \mathcal{P}\right]^{a i} K_{a}{ }^{b}{ }^{i}
\end{aligned}
$$

This latter equation is merely an identity, as expected from reparametrization invariance. The first equation can be used to express the linearized equations of motion, about a solution of the equations of motion, in the form

$$
\widetilde{\nabla}_{a}\left[\delta_{\perp} \mathcal{P}\right]^{a i}-\left[\delta_{\perp} \mathcal{P}\right]^{a b} K_{a b}^{i}=0
$$

Note the apparent similarity with the equations of motion expressed in the form (44). The usefulness of this expression is that in general, the variation of the momentum density is easier to calculate than the variation of the Euler-Lagrange derivative.

The variation of the total linear momentum is then simply

$$
\delta_{\perp} P^{\mu}(\Sigma)=\int_{\partial m} \eta_{a} \delta_{\perp} \mathcal{P}^{a \mu}
$$

Again, it should be emphasized that this is much easier than a direct variation of $P^{\mu}(\Sigma)$.

In order to illustrate this approach, and to give a simple application, let us consider a DNG object. The linear momentum density is given by Eq. (51). We can use Eqs. (62), (47), together with the first Gauss-Weingarten deformation equation (see Ref. [19])

$$
\delta_{\perp} e^{\mu}{ }_{a}=K_{a b}{ }^{i} \Phi_{i} e^{\mu b}+\left(\widetilde{\nabla}_{a} \Phi^{i}\right) n^{\mu}{ }_{i}
$$

so that

$$
\delta_{\perp} \mathcal{P}^{a \mu}=-\mu \sqrt{-\gamma}\left[\left(K_{i} \gamma^{a b}-K^{a b}{ }_{i}\right) \Phi^{i} e^{\mu}{ }_{b}+\left(\widetilde{\nabla}^{a} \Phi^{i}\right) n^{\mu}{ }_{i}\right]
$$


We read off the projections,

$$
\begin{gathered}
{\left[\delta_{\perp} \mathcal{P}\right]^{a b}=-\mu \sqrt{-\gamma}\left(K_{i} \gamma^{a b}-K^{a b}{ }_{i}\right) \Phi^{i}} \\
{\left[\delta_{\perp} \mathcal{P}\right]^{a i}=-\mu \sqrt{-\gamma} \widetilde{\nabla}^{a} \Phi^{i} .}
\end{gathered}
$$

Recalling that, for a DNG object, $\mathcal{E}_{i}=-\mu K_{i}$, and substituting in Eq. (107), we find

$$
\begin{aligned}
\left(\tilde{\delta}_{\perp} K^{i}\right) & =-K^{j} \Phi_{j} K^{i}-\widetilde{\nabla}_{a}\left(\widetilde{\nabla}^{a} \Phi^{i}\right)+\left(K_{j} \gamma^{a b}-K^{a b}{ }_{j}\right) \Phi^{j} K_{a b}{ }^{i} \\
& =-\widetilde{\nabla}_{a}\left(\widetilde{\nabla}^{a} \Phi^{i}\right)-K_{a b}{ }^{i} K^{a b}{ }_{j} \Phi^{j},
\end{aligned}
$$

which agrees with the expression derived e.g. in Ref. [19] (see also [33, 37, 38]).

Let us now confirm that Eq. (108) is in fact an identity. Substitution of the projections gives

$$
K^{i}\left(\widetilde{\nabla}^{b} \Phi_{i}\right)=\widetilde{\nabla}_{a}\left[\left(K_{i} \gamma^{a b}-K^{a b}{ }_{i}\right) \Phi^{i}\right]+\left(\widetilde{\nabla}^{a} \Phi^{i}\right) K_{a}{ }^{b}{ },
$$

and this expression is identically zero, as follows from the Codazzi-Mainardi integrability condition, Eq. (93).

This example renders transparent the advantages of this approach. Rather than dealing with the variation of the extrinsic curvature, all one needs here is the variation of the intrinsic geometry of the worldvolume.

What about the second variation parallel to the worldvolume, $\delta_{\|}(\delta S)$ ? As expected from reparametrization invariance, it adds nothing new. We show this explicitly in Appendix B for a DNG brane.

\section{Einstein-Hilbert action with surface term}

In this section, we consider the addition of a surface term to the Einstein-Hilbert brane action we have briefly described at the end of Sect. 6,

$$
S=S_{E H}+S_{\partial m}
$$

where the first term is defined in Eq. (98), and

$$
S_{\partial m}=2 \beta \int_{\partial m} \sqrt{h} \kappa
$$

and $\kappa$ is the trace of the extrinsic curvature $\kappa_{A B}$ of the spacelike boundary provided by $\Sigma_{(i)}$ and $\Sigma_{(f)}$ embedded in $m$.

An analogous term is added to the Einstein-Hilbert action in general relativity in order that the variational principle applied to the action yield the Einstein equations in a bounded region subject to the boundary condition that the metric induced on the boundary is fixed and no more [22, 23]. Technically, the variation of the boundary term precisely cancels normal derivatives of the variation of the metric tensor on the boundary, which occur in the variation of the Einstein Hilbert action. In general relativity this term is 
diffeomorphism invariant. Here, it is also Poincaré invariant. Interestingly enough, the particle analogue mentioned in Sect. 6 is incomplete in this respect. Whereas the original lagrangian $L_{0}(x, \dot{x})=(1 / 2) \dot{x}^{2}$ is invariant under translations, the lagrangian $L(x, \ddot{x})=$ $-(1 / 2) x \ddot{x}$ is not and so Noether's theorem cannot be applied directly to it. The boundary term is necessary to restore the translation invariance of the problem.

The variation of Eq. (117) poses new technical difficulties. In general relativity, the dynamical variables to be varied are the spacetime metric coefficients. In the present context, we need to vary the embedding functions describing the worldvolume. Fortunately, the relevant formalism has been developed in Refs. 31, 39], for the case of a timelike boundary. Its adaptation to the case of interest here requires only some minor sign modifications.

The problem is simplified by treating the boundary of the worldvolume as two embedded spacelike surfaces in the background Minkowski space-time described by Eq. (田). We denote the normals to $\partial m$ in spacetime by $m_{I}^{\mu}(I, J, \cdots=0,1,2, \cdots, N-D)$. It is also convenient to exploit a normal basis which is adapted to the worldvolume $m$ : we choose the basis $m^{\mu I}=\left\{\eta^{\mu}, n^{\mu}{ }_{i}\right\}$, supplementing the normals $n^{\mu}{ }_{i}$ to $m$ in spacetime with $\eta^{\mu}=e^{\mu}{ }_{a} \eta^{a}$, the normal to the boundary which is tangent to the worldvolume. Let $L_{A B}{ }^{I}$ represent the extrinsic curvatures associated with the embedding $X$. With respect to the adapted basis, $L_{A B}{ }^{i}=K_{a b}{ }^{i} \epsilon^{a}{ }_{A} \epsilon_{B}^{b}=K_{A B}{ }^{i}$, and $L_{A B}{ }^{0}=\kappa_{A B}$. (For details see [3], 39].

Let us examine an arbitrary deformation of $\partial m$ in spacetime. If $\partial m$ is closed (as we assume), we need only consider a normal deformation of $\partial m$. Let us first expand

$$
\delta_{\bar{\perp}} X^{\mu}=\Phi^{I} m^{\mu}{ }_{I}=\psi \eta^{\mu}+\phi^{i} n^{\mu}{ }_{i} .
$$

We use the symbol $\perp$ to distinguish this normal variation, which includes a variation along $\eta^{\mu}$, from the worldvolume normal variation used earlier. We have also expressed the variation with respect to the adapted basis, so that $\Psi^{0}=\psi, \Psi^{i}=\psi^{i}$.

We now exploit the formalism developed in Ref. [39] to express the induced normal variations of $h_{A B}$ and $L_{A B}{ }^{I}$ as follows:

$$
\begin{aligned}
\delta_{\perp} h_{A B} & =2 L_{A B}{ }^{I} \Phi_{I} \\
\delta_{\bar{\perp}} L_{A B}{ }^{I} & =-\hat{\mathcal{D}}_{A} \hat{\mathcal{D}}_{B} \Phi^{I}+L_{A}{ }^{C I} L_{C B J} \Phi^{J}+\hat{\gamma}_{J}^{I} L_{A B}{ }^{J} .
\end{aligned}
$$

Here $\mathcal{D}_{A}$ is the covariant derivative compatible with $h_{A B}$, and $\hat{\mathcal{D}}_{A}$ its extension that is covariant under rotations of normals to $\partial m$ in spacetime. We need to compute the variation for the component $I=0$ in this last expression. The boundary term is not covariant under rotations of the normals, $m_{I}^{\mu}: \kappa_{A B}$ is the extrinsic curvature which corresponds to $\eta^{\mu}$, there is no rotational arbitrariness here. For this reason, the appropriate deformation operator we apply to $\kappa$ is $\delta_{\bar{I}}$ and not the manifestly rotationally covariant object $\tilde{\delta}_{\bar{\perp}}$ [31. This implies the necessity to restore the deformation connection $\hat{\gamma}^{I}{ }_{J}$ on the right hand side of Eq. (120). This would spell disaster if we needed to evaluate all of $\hat{\gamma}_{I J}$. However, we only require $\hat{\gamma}_{0 i}$ which is well defined on $\partial m$. We then find [39]

$$
\begin{aligned}
\delta_{\perp} \kappa & =-\mathcal{D}_{A} \mathcal{D}^{A} \psi+\left(K^{A i} K_{A i}-\kappa^{A B} \kappa_{A B}-h^{A B} K_{A B}{ }^{i} K_{a b i} \eta^{a} \eta^{b}\right) \psi \\
& -\mathcal{D}_{A}\left(K^{i A} \phi_{i}\right)+\left(\eta^{a} h^{A B} K_{A B}{ }^{i}-\epsilon^{a A} K_{A}{ }^{i}\right) \tilde{\nabla}_{a} \phi_{i}-\kappa^{A B} K_{A B}{ }^{i} \phi_{i} .
\end{aligned}
$$


To arrive at this expression we have used the fact that $\hat{\gamma}^{0 i}=-\eta^{a} \eta^{b} K_{a b}^{i} \psi+\eta^{a} \widetilde{\nabla}_{a} \phi^{i}$ and we have introduced the notation, $K_{A}{ }^{i}=\eta^{a} \epsilon_{A}^{b} K_{a b}^{i}$. Some simplification is possible. Let us begin with the terms linear in $\psi$. We can use the contracted Gauss-Codazzi equation to obtain,

$$
K^{A i} K_{A i}-h^{A B} K_{A B}{ }^{i} K_{a b i} \eta^{a} \eta^{b}=-\eta^{a} \eta^{b} \mathcal{R}_{a b}
$$

In addition, we have

$$
\left(\eta^{a} h^{A B} K_{A B}^{i}-\epsilon^{a A} K_{A}^{i}\right) \widetilde{\nabla}_{a} \phi_{i}=\eta_{a}\left(K^{i} \gamma^{a b}-K^{a b i}\right) \widetilde{\nabla}_{b} \phi_{i}
$$

Inserting these partial results into Eq. (121), we obtain

$$
\begin{aligned}
\delta_{\perp} \kappa & =-\mathcal{D}_{A} \mathcal{D}^{A} \psi-\left(\kappa^{A B} \kappa_{A B}+\eta^{a} \eta^{b} \mathcal{R}_{a b}\right) \psi \\
& -\mathcal{D}_{A}\left(K^{i A} \phi_{i}\right)+\eta_{a}\left(K^{i} \gamma^{a b}-K^{a b i}\right) \widetilde{\nabla}_{b} \phi_{i}-\kappa^{A B} K_{A B}{ }^{i} \phi_{i}
\end{aligned}
$$

We have now all the ingredients needed for the calculation of the normal variation of the boundary action (117). We neglect total derivatives, and we find

$$
\begin{aligned}
\delta_{\perp} S_{\partial m} & =2 \beta \int_{\partial m} \sqrt{h}\left\{\left[\kappa^{2}-\kappa_{A B} \kappa^{A B}-\eta^{a} \eta^{b} \mathcal{R}_{a b}\right] \psi\right. \\
& \left.+\left(h^{A B} \kappa-\kappa^{A B}\right) K_{A B}{ }^{i} \phi_{i}+\eta_{a}\left(K^{i} \gamma^{a b}-K^{a b i}\right) \widetilde{\nabla}_{b} \phi_{i}\right\}
\end{aligned}
$$

To establish contact with the bulk variation, we must identify $\psi=\eta^{a} \Phi_{a}$. The total boundary contribution, obtained by summing this expression with the second line of Eq. (102), therefore does not contain any term involving derivatives of the $\Phi^{i}$.

Let us consider now a translation of the boundary in the background space-time. The linear momentum associated with this contribution is then given by

$$
p^{\mu}(\Sigma)=2 \beta \int_{\Sigma} \sqrt{h}\left\{\eta^{\mu}\left[\kappa_{A B} \kappa^{A B}-\kappa^{2}+\eta^{a} \eta^{b} \mathcal{R}_{a b}\right]+\eta^{a} \mathcal{R}_{a b} e^{\mu b}+K_{A B}^{i}\left(h^{A B} \kappa-\kappa^{A B}\right) n^{\mu}{ }_{i}\right\} .
$$

It should be noticed that the surface momentum has an additional normal component. The linear momentum for the total action (116) using Eq. (102), becomes

$$
P^{\mu}(\Sigma)+p^{\mu}(\Sigma)=\beta \int_{\Sigma} \sqrt{h}\left\{\eta^{\mu}\left[\kappa_{A B} \kappa^{A B}-\kappa^{2}+\eta^{a} \eta^{b} \mathcal{G}_{a b}\right]+K_{A B}^{i}\left(h^{A B} \kappa-\kappa^{A B}\right) n^{\mu}{ }_{i}\right\} .
$$

Note that this expression vanishes identically for a string, since for $D=2$, we have $\mathcal{G}_{a b}=0$, and moreover in the degenerate case of a one dimensional boundary, $k_{A B}=h_{A B} k$.

Consider now an infinitesimal Lorentz transformation. The boundary contribution to the angular momentum is given by

$$
m^{\mu \nu}(\Sigma)=\frac{1}{2} \int_{\Sigma}\left\{\tilde{p}^{\mu} X^{\nu}+2 \beta \sqrt{h} \eta_{a}\left(K^{i} \gamma^{a b}-K^{a b i}\right) n^{\mu}{ }_{i} e_{b}^{\nu}-(\mu \leftrightarrow \nu)\right\}
$$

where the quantity $\tilde{p}^{\mu}$ in the first term is the integrand appearing in Eq.(124). 
The second term is what is needed to cancel the offending term in the angular momentum for the bulk Einstein-Hilbert action, in Eq. (103), so that the angular momentum for the total action is now

$$
M^{\mu \nu}(\Sigma)+m^{\mu \nu}(\Sigma)=\frac{1}{2} \int_{\Sigma}\left[\Pi^{\mu} X^{\nu}-(\mu \leftrightarrow \nu)\right]
$$

where we denote with $\Pi^{\mu}$ the integrand of the total linear momentum (125).

In conclusion, the same surface term that is appropriate to lower the order of the boundary conditions, also cancels the kinematic term in the angular momentum density.

\section{Discussion}

In this paper, we have presented a new approach to the derivation of the linear and angular momentum for a brane propagating in Minkowski spacetime based on the worldvolume geometry. We have considered a large class of brane actions, depending on the intrinsic and as well on the extrinsic geometry of the worldvolume, up to a first derivative of the extrinsic curvature. The generalization to a more general action is straightforward, following the guidelines given in the paper.

This analysis may be extended in a straightforward way to treat the corresponding conserved quantities for a brane propagating on a background spacetime possessing Killing vector fields.

A particular simple case we have not discussed explicitly here is the degenerate case of a point object described by a higher order action. We will discuss this case elsewhere.

It would also be interesting to apply this geometrical approach to supersymmetric branes 41]. While the geometry of such objects is well understood, to our knowledge, the geometry of deformations of superembedded surfaces remains to be developed.

\section{Acknowledgements}

We have received partial support from CONACyT grant no. 211085-5-0118PE. Our thanks to Charles Torre for useful suggestions. 


\section{Appendix A}

In this section, we consider the more general case of an action that depends also on first derivatives of the extrinsic curvature,

$$
S_{(h o)}[Y]=\int_{m} \sqrt{-\gamma} L\left(\gamma^{a b}, K_{a b}^{i}, \widetilde{\nabla}_{a} K_{b c}{ }^{i}\right) .
$$

We can recycle the results of Sect. 5, for the variation with respect to the first arguments, that will not be repeated here. The new part is

$$
\delta_{\perp} S_{(h o)}=\int_{m} L_{i}^{a b c} \tilde{\delta}_{\perp} \widetilde{\nabla}_{a} K_{b c}^{i}
$$

where we have defined

$$
L_{i}^{a b c}=\frac{\partial L}{\partial \widetilde{\nabla}_{a} K_{b c}{ }^{i}} .
$$

Note that, as a consequence of the Codazzi-Mainardi integrability condition, and the symmetry of $K_{a b}{ }^{i}$, we have the symmetry property

$$
\widetilde{\nabla}_{a} K_{b c}{ }^{i}=\widetilde{\nabla}_{(a} K_{b c)}{ }^{i}
$$

from which it follows that

$$
L_{i}^{a b c}=L_{i}^{(a b c)}{ }_{i}
$$

In order to evaluate Eq. (129), we need to commute $\tilde{\delta}_{\perp}$ with $\tilde{\nabla}_{a}$. To do this, we need the following expressions (see also [19])

$$
\begin{aligned}
\delta_{\perp} \gamma_{a b}{ }^{c} & =\gamma^{c d}\left[\nabla_{a}\left(K_{b d}{ }^{i} \Phi_{i}\right)+\nabla_{b}\left(K_{a d}{ }^{i} \Phi_{i}\right)-\nabla_{d}\left(K_{a b}{ }^{i} \Phi_{i}\right)\right], \\
\delta_{\perp} \omega_{a}{ }^{i j} & =K_{a}{ }^{b i} \widetilde{\nabla}_{b} \Phi^{j}-K_{a}{ }^{b j} \widetilde{\nabla}_{b} \Phi^{i} .
\end{aligned}
$$

Now, it is a direct computation to obtain that the contribution of (129) to the boundary term of the normal variation of the action is given by,

$$
\begin{aligned}
\delta_{\perp} S_{(h o)} & =\int_{\partial m} \sqrt{h} \eta_{a}\left[-\left(\widetilde{\nabla}_{b} \widetilde{\nabla}_{c} L^{a b c}{ }_{i}\right) \Phi^{i}+\left(\widetilde{\nabla}_{b} L^{a b c}{ }_{i}\right) \widetilde{\nabla}_{c} \Phi^{i}-L^{a b c} \widetilde{\nabla}_{b} \widetilde{\nabla}_{c} \Phi^{i}\right. \\
& \left.-L^{b c d}{ }_{i} K_{b c}{ }^{j} K^{a}{ }_{d j} \Phi^{i}+3 L^{b c d}{ }_{j} K_{b c i} K_{d}{ }^{a j} \Phi^{i}-3 L_{j}^{a b c} K_{b}{ }^{d j} K_{c d i} \Phi^{i}\right] .
\end{aligned}
$$

If this seems complicated, well the Euler-Lagrange derivative is worse.

For an infinitesimal translation, we obtain that the total contribution to the momentum density is

$$
\begin{aligned}
\mathcal{P}^{a \mu} & =\sqrt{-\gamma}\left\{\left[L_{(h o)} \delta_{d}^{a}-L_{i}^{a b c}\left(\widetilde{\nabla}_{b} K_{c d}{ }^{i}\right)+\left(\widetilde{\nabla}_{b} L^{a b c}{ }_{i}\right) K_{c d}{ }^{i}\right] e^{\mu d}\right. \\
& +\left[-\left(\widetilde{\nabla}_{b} \widetilde{\nabla}_{c} L^{a b c}{ }_{i}\right)-L^{b c d}{ }_{i} K_{b c}{ }^{j} K^{a}{ }_{d j}+3 L^{b c d}{ }_{j} K_{b c i} K_{d}{ }^{a j}\right. \\
& \left.\left.-2 L^{a b c}{ }_{j} K_{b}{ }^{d j} K_{c d i}\right] n^{\mu i}\right\} .
\end{aligned}
$$


The contribution to the angular momentum density is

$$
\begin{aligned}
\mathcal{M}^{a \mu \nu}=\frac{1}{2}\left\{\mathcal{P}^{a \mu} X^{\nu}\right. & +\sqrt{-\gamma}\left[\left(\widetilde{\nabla}_{b} L^{a b c}{ }_{i}\right) n^{\mu i} e^{\nu}{ }_{c}-2 L_{i}^{a b c} K_{c d}{ }^{i} e^{\mu d} e^{\nu}{ }_{b}\right. \\
& \left.\left.+L^{a b c}{ }_{i} K_{b c}{ }^{j} n^{\mu i} n^{\nu}{ }_{j}\right]-(\mu \leftrightarrow \nu)\right\} .
\end{aligned}
$$

We note that in this expression all of the bivectors enter.

\section{Appendix B}

In this appendix, we show explicitly that, as stated at the end of Sect. 7, the vanishing of the second variation parallel to the worldsheet, $\delta_{\|}(\delta S)$ results in mere identities.

The parallel variation of Eq. (25) gives

$$
\delta_{\|}(\delta S)=\epsilon_{\mu} \int_{m} \nabla_{a}\left[\sqrt{-\gamma} \Phi^{a} \mathcal{E}^{i} n^{\mu}{ }_{i}+\delta_{\|} \mathcal{P}^{a \mu}\right],
$$

where we have used Eq. (19), valid for any worldvolume scalar density of weight one. We decompose $\delta_{\|} \mathcal{P}^{a \mu}$ in terms of its worldvolume projections,

$$
\delta_{\|} \mathcal{P}^{a \mu}=\left[\delta_{\|} \mathcal{P}\right]^{a b} e^{\mu}{ }_{b}+\left[\delta_{\|} \mathcal{P}\right]^{a i} n^{\mu}{ }_{i} .
$$

Substituting into Eq. (137), and using the Gauss-Weingarten equations (40),(41), we find the parallel analogue of Eqs. (107), (108),

$$
\begin{gathered}
\nabla_{a}\left(\sqrt{-\gamma} \Phi^{a} \mathcal{E}^{i}\right)=-\widetilde{\nabla}_{a}\left[\delta_{\|} \mathcal{P}\right]^{a i}+\left[\delta_{\|} \mathcal{P}\right]^{a b} K_{a b}{ }^{i}, \\
\sqrt{-\gamma} \Phi^{a} \mathcal{E}^{i} K_{a}{ }^{b}{ }_{i}=-\widetilde{\nabla}_{a}\left[\delta_{\|} \mathcal{P}\right]^{a b}-\left[\delta_{\|} \mathcal{P}\right]^{a i} K_{a}{ }^{b}{ }_{i} .
\end{gathered}
$$

Both these equations are mere identities. Let us confirm this for the special case of a DNG object. Using the parallel deformation Gauss-Weingarten equation (see [40]),

$$
\delta_{\|} e^{\mu}{ }_{a}=\left(\nabla_{a} \Phi^{b}\right) e^{\mu}{ }_{b}+K_{a b}{ }^{i} \Phi^{b} n^{\mu}{ }_{i},
$$

we have that the parallel variation of Eq. (51) gives

$$
\delta_{\|} \mathcal{P}^{a \mu}=-\mu \sqrt{-\gamma}\left[\left(\nabla_{c} \Phi^{c} \gamma^{a b}-\nabla^{b} \Phi^{a}\right) e^{\mu}{ }_{b}-K^{a b i} \Phi_{b} n^{\mu}{ }_{i}\right],
$$

so that the worldvolume projections are

$$
\begin{gathered}
{\left[\delta_{\|} \mathcal{P}\right]^{a b}=-\mu \sqrt{-\gamma}\left(\nabla_{c} \Phi^{c} \gamma^{a b}-\nabla^{b} \Phi^{a}\right),} \\
{\left[\delta_{\|} \mathcal{P}\right]^{a i}=\mu \sqrt{-\gamma} K^{a b i} \Phi_{b} .}
\end{gathered}
$$

Substituting into Eq. (138) gives

$$
\nabla_{b}\left(\Phi^{b} K^{i}\right)=\nabla_{a}\left[K^{a b i} \Phi_{b}\right]+\left(\nabla_{c} \Phi^{c} \gamma^{a b}-\nabla^{b} \Phi^{a}\right) K_{a b}{ }^{i} .
$$


Using the contracted Codazzi-Mainardi integrability condition, Eq. (93), one can easily verify that this equation is satisfied identically. On the other hand, substitution of the projections into Eq. (139) gives

$$
\Phi^{a} K_{i} K_{a}{ }^{b i}=\Phi_{c} K^{a c i} K_{a}{ }^{b}{ }_{i}-\nabla_{a}\left(\nabla_{c} \Phi^{c} \gamma^{a b}-\nabla^{b} \Phi^{a}\right)
$$

This equation can be seen to vanish identically as well. This requires the contracted GaussCodazzi equation (58), for the lefthand side together with the first term on the right hand side, and the Ricci identity for the remaining two terms,

$$
\left(\nabla_{a} \nabla_{b}-\nabla_{b} \nabla_{a}\right) \Phi^{b}=-\mathcal{R}_{a b} \Phi^{b}
$$




\section{References}

[1] P.A.M. Dirac, Proc. Royal Soc. A 268 (1962), 57.

[2] P.A. Collinson, J.F.L. Hopkinson, and R.W. Tucker, Nucl. Phys. B 100 (1975), 157.

[3] J. Nambu, Copenhagen Summer Symposium (1970), unpublished; T. Goto, Prog. Theor. Phys. 46 (1971), 1560.

[4] M. Green, J. Schwarz and E. Witten, Superstring Theory (Cambridge University Press, 1987).

[5] C.P. Bachas, Lectures on D-branes (hep-th/9806199).

[6] H. Nicolai, and R. Helling, Supermembranes and M(atrix) Theory, (hep-th/9809103).

[7] A. Polyakov, Nucl. Phys. B 268 (1986), 406; H. Kleinert, Phys. Lett. B 174 (1986), 335.

[8] P.B. Canham, J. Theor. Biol 26 (1970), 61; W. Helfrich, Z. Naturforsch. 28c (1973), 693.

[9] K. Maeda, and N. Turok, Phys. Lett. B 202 (1988), 376; R. Gregory, Phys. Lett. B 206 (1988), 206; Phys. Rev. D 43 (1991), 520; D. Garfinkle and R. Gregory, Phys. Rev. D 41 (1990), 1889; R. Gregory, D. Haws, and D. Garfinkle, Phys. Rev. D 42 (1990), 343; V. Silveira, and M.D. Maia, Phys. Lett. A 174 (1993), 280; H. Arodź, Nucl. Phys. B 450 (1995) 174; ibid. 450 (1995) 189.

[10] B. Carter and R. Gregory, Phys. Rev. D 51 (1995), 5839.

[11] M. Anderson, F. Bonjour, R. Gregory and J. Stewart, Phys. Rev. D 56 (1997) 8014.

[12] A.M. Polyakov, Gauge Fields and Strings (Harwood Academic Publishers, 1987).

[13] J. Ambjorn, B. Durhuus, and T. Jonsson, Quantum Geometry: A statistical field approach (Cambridge University Press, 1997).

[14] P.B. Wiegmann, Nucl. Phys. B 323 (1989) 330.

[15] J. Scherk, Rev. Mod. Phys. 47 (1975), 123.

[16] P.S. Letelier, Phys. Rev. D 18 (1978), 359.

[17] V. Iyer, and R. Wald, Phys. Rev. D 50 (1994), 846.

[18] B. Carter, in Recent Developments in Gravitation and Mathematics ( Proc. 2nd Mexican School on Gravitation and Mathematical Physics, Tlaxcala 1996) eds. A. Garcia et al. (Science Network Publishing, Konstanz 1997) (hep-th/9705172). 
[19] R. Capovilla and J. Guven, Phys. Rev. D 51 (1995), 6736.

[20] T. Regge, and C. Teitelboim, in Proc. Marcel Grossmann, Trieste 1975, p.77.

[21] S. Deser, F.A.E. Pirani, and D.C. Robinson, Phys. Rev. D 14 (1976), 3301.

[22] J.W. York, Phys. Rev. Lett. 28 (1972), 1082.

[23] S.W. Hawking, in General Relativity: An Einstein Centenary Survey eds. S.W. Hawking, and W. Israel (cambridge University press, 1979).

[24] P.S. Letelier, Phys. Rev. D 41 (1990), 1333.

[25] B. Boisseau, and P.S. Letelier, Phys. Rev. D 46 (1992), 1721.

[26] B. Carter, Class. Quantum Grav. 11 (1994), 2677.

[27] D.H. Hartley and R.W. Tucker, in Geometry of Low-Dimensional Manifolds: 1, London Mathematical Society Lecture Note Series 150, ed. by S.K. Donaldson and C.B. Thomas (Cambridge University Press, Cambridge, 1990).

[28] I.M. Anderson, in Mathematical Aspects of Classical Field Theory, eds. M. Gotay, J. Marsden, and V. Moncrief, Contemp. Math. 132 (1992), 51.

[29] I.M. Anderson, and C. Torre, Phys. Rev. Lett. 77 (1996), 4109.

[30] C. Torre, in Recent Developments in Gravitation and Mathematics ( Proc. 2nd Mexican School on Gravitation and Mathematical Physics, Tlaxcala 1996) eds. A. Garcia et al. (Science Network Publishing, Konstanz 1997).

[31] R. Capovilla and J. Guven, Phys. Rev. D 55 (1997), 2388.

[32] C. Aragone, and S. Deser, Nucl. Phys. B92 (1975), 327.

[33] J. Guven, Phys. Rev. D 48 (1993), 5562.

[34] L.D. Landau, and E.M. Lifschitz, Theory of Elasticity, 2nd ed., revised and enlarged (Pergamon, New York, 1970).

[35] T.L. Curtright, G.I. Ghandour, C.B. Thorn, and K. Zachos, Phys. Rev. Lett. 57 (1986), 799.

[36] J. Garriga, and A. Vilenkin, Phys. Rev. D, 47 (1993), 3265.

[37] B. Carter, Phys. Rev. D , 48 (1994), 4835.

[38] A.L. Larsen, and V. Frolov, Nucl. Phys. B 414 (1994), 129.

[39] R. Capovilla and J. Guven, Phys. Rev. D 57 (1998), 5158. 
[40] R. Capovilla and J. Guven, Class. Quant. Grav. 12 (1995), L107.

[41] I.A. Bandos, D. Sorokin, M. Tonin, P. Pasti, and D. Volkov, Nucl. Phys. B 446 (1995), 79; P.S. Howe, O. Raetzel, and E. Sezgin, JHEP 08 (1998), 59; E. Sezgin, Topics in M-theory (hep-th/9809204). 